\title{
Administration of Fresh Juice of Tinospora cordifolia Decreases Levels of Urinary Markers of Peroxisome Proliferator-Activated Receptors in Hyperlipidemic Patients
}

\author{
Amey R. Shirolkar ${ }^{1}$ and Rajesh Dabur ${ }^{1,2 *}$ \\ ${ }^{1}$ Department of Biochemistry, National Research Institute of Basic Ayurvedic Sciences, Pune, Maharashtra-411038, INDIA. \\ ${ }^{2}$ Department of Biochemistry, Maharshi Dayanand University, Rohtak, Haryana-124001, INDIA.
}

\begin{abstract}
Objective: Tinospora cordifolia (Willd.) Miers juice (TCJ) is known for its anti-hyperlipidemic properties. This study was aimed to validate the efficacy of TCJ to activate PPAR $\alpha$ in vivo in hyperlipidemic (HPL) patients. Materials and Methods: HPL $(n=20)$ and healthy $(n=22)$ volunteers were enrolled in the study. HPL patients were treated with TCJ for 14 days. The fasting urine and blood samples were collected on 0 and $14^{\text {th }}$ day for analysis. The blood samples were used for biochemical analysis. Urine samples were subjected to LC-ESI-QTOFMS for the analysis of PPAR- $\alpha$ markers. Results: Treatment with TCJ depleted cholesterol (TC-24\%), triglycerides (TG-28\%) and low density lipoprotein (LDL$14 \%)$ levels and increased high density lipoprotein (HDL) $(p<0.05)$ levels in HPL. The QTOFMS data revealed increased urinary excretions of pantothenic acid (5.99 fold), hippuric acid ( $<15.0$ fold), acylcarnitines $(<2.0$ folds) and branched chain amino acids (BCAA) in HPL patients which significantly depleted $(p<0.01)$ after TCJ treatment. Two new acylcarnitines, L-hexanoylcarnitine and 4-hydroxyisovaleric acid observed in HPL patients were depleted ( $>2.0$ fold) after treatment with TCJ. The other biomarkers of $\mathrm{HPL}$, ursodeoxycholic acid and chenodeoxycholic acid were also depleted ( $>4.0$ fold) after TCJ treatment. Conclusion: The present data clearly depicted the regulatory effects of TCJ on the lipid metabolism. It emphasize that TCJ activates PPAR- $\alpha$ in vivo that decreased the urinary levels of PPAR $\alpha$ biomarkers.
\end{abstract}

Key words: Tinospora cordifolia, Urinary biomarkers, LC-ESI-QTOFMS, PPAR-alpha, Fatty acids $ß$ oxidation, Oxidative stress.

\section{INTRODUCTION}

Tinospora cordifolia (Willd.) Miers (Menispermaceae) is a well known medicinal plants reported by various ancient literatures. Scientific studies have been carried out by various labs across the globe also proved the medicinal values of the plant. Medicinal properties, chemical constituents and clinical studies of this plant are well documented. ${ }^{1}$ In addition, ancient Indian literature, Ayurveda and others reported T. cordifolia for its rejuvenating properties. ${ }^{2,3}$ The plant is very popular for its immunomodulatory properties and to increase the platelets. ${ }^{4}$ A number of bioactive compounds, including berberine, cordifoliosides, tinosporoids etc have reported from the plant. ${ }^{5}$ Different compounds from the plant have been reported for different type of medicinal properties. ${ }^{1,6}$ For example, berberine is reported as anti-hyperlipidemic agent having different mechanisms than statins ${ }^{7}$ whereas palmatine was reported for antidiabetic properties. ${ }^{8}$
Submission Date : 19-10-2015 Revision Date : 29-02-2016 Accepted Date : 14-03-2016

DOI: 10.5530/ijper.50.3.19 Correspondence: Dr. Rajesh Dabur, Department of Biochemistry, Maharshi Dayanand University, Rohtak, Haryana-124001, INDIA. Phone: +911262-393070 E-mail: rajeshdabur@yahoo. com

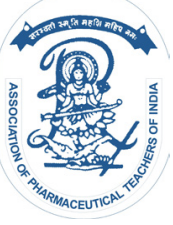

www.ijper.org 
Recently, crude extracts of the plant have been reported to have in vitro anti-diabetic properties by up-regulation of PPAR $\alpha$ through unknown mechanisms. ${ }^{9}$ A number of in vivo studies have already been conducted to validate the in vitro efficacy of the plant extracts. ${ }^{10}$ However, it is well known fact that in vitro mechanism of drugs cannot be correlated with in vivo mechanism due to the complex phenomenon which starts from gut absorption of compounds to their catabolism. Therefore, current study was undertaken to explore and validate the in vivo antihyperlipidemic mechanism of TCJ. HPLC-Q-TOF-MS was used to explore urinary markers of hyperlipidemia. It was effectively used to predict and validate the levels of biomarkers of hyperlipidemia in human urine earlier reported in mouse models. ${ }^{11}$

\section{MATERIALS AND METHODS}

\section{Chemicals}

Standards of pantothenic acid, acylcarnitines, amino acids, ursodeoxycholic acid and solvents i.e. water, acetonitrile, formic acid, were purchased from Sigma (St. Louis, MO). Internal and external calibrates and standards were purchased from Agilent Technologies. All other reagents used in the study were LC-MS grade (Sigma).

\section{Study subjects}

The study protocol was approved by the Ethics Committee of Padmashree Dr. D.Y. Patil Medical College, Akurdi, Pune, India. A written consent was taken from every individual before entering into the study. Volunteer males having hyperlipidemia were selected for the study from the local areas. The protocols for this study were designed in accordance with diagnostic criteria for hypertriglyceridemia by the Indian Medical Association. Clinical features of the hypertriglyceridemia used for screening were TG $>300 \mathrm{mg} / \mathrm{dL}$, blood pressure $>160 / 90 \mathrm{~mm} \mathrm{Hg}$ and $\mathrm{TC}>130-230 \mathrm{mg} / \mathrm{dL}$. The basic data included sex, age, body mass index (BMI), alcohol and tobacco use, and diabetes mellitus. Physical examination and laboratory tests were performed within $2 \mathrm{hrs}$ of admission. TG, TC and serum lipase liver enzymes levels at the time of admission were measured. The severity of the patient condition was classified according to the Ranson score, Glasgow score and Apache II score. Exclusion criteria of patients included body mass index $<30 \mathrm{~kg} / \mathrm{m}^{2}$, cardiovascular disease, diabetes mellitus, blood pressure $<140 / 90 \mathrm{~mm} \mathrm{Hg}, \mathrm{TC}<7.5 \mathrm{mmol} / \mathrm{L}$ or use of lipid-lowering agents, antihypertensive agents. Therefore, only the patients with mild hyperlipidemia were included into the study. Urine and plasma samples were collected in fasting conditions, centrifuged at $6000 \mathrm{~g}$ for $10 \mathrm{~min}$ and stored at $-80^{\circ} \mathrm{C}$ until use.

\section{Drug preparation}

Spot identified fresh stems of T. cordifolia plants were collected in September 2011 from garden of National Research Institute of Basic Ayurvedic Sciences, CCRAS (Department of AYUSH), Nehru Garden, Kothrud, Pune. The voucher specimens (No. 207) were kept at the medicinal plant museum of the Institute. The stems were washed with $\mathrm{RO}$ water, crushed and kept in RO water (20:100 wt: vol of the stem and water) overnight at room temperature. TCJ was prepared under the guidance of an Ayurvedic physician.

\section{Study design}

Forty two volunteers $\{20 \mathrm{HPL}$ and 22 healthy (HLT) $\}$ of 36-40 years age group (38 \pm 2.0 year mean age) were enrolled in the study. All volunteers have asked to maintain their usual diet throughout the study and not to take any medicine without consultation. After one week pre-watch analysis period, only the persons who had consistent patterns of TG and TC levels were included as hyperlipidemic patients. Out of 42, $10 \mathrm{HPL}$ and 11 HLT volunteers were given $100 \mathrm{ml}$ TCJ early in the morning with an empty stomach for 14 days in the presence of a physician. Other half volunteers were given an orange juice and kept as a placebo control. Two HLT volunteers have been dropping out of the study due their inconsistent presence. Plasma and urine sample for analysis were collected at 0 days (after 7 day observation) and on the $14^{\text {th }}$ day after administration of TCJ.

\section{Hematology and blood biochemistry}

Blood samples were collected from the antecubital fosse into cold tubes containing EDTA and reduced glutathione and centrifuged immediately at $1000 \mathrm{~g}$ for $10 \mathrm{~min}$ at $4^{\circ} \mathrm{C}$. The plasma samples were added with butylated hydroxytoluene $(40 \mu \mathrm{g} / \mathrm{ml}$ plasma) to protect them from oxidation and stored at $-80^{\circ} \mathrm{C}$ until analysis. Serum TG, TC, LDL, HDL, creatinine, urea, SGOT, SGPT, total proteins, albumin and blood glucose levels in plasma were measured using auto analyzer D-280 (Sinnowa, China Republics). Hematology was done using an automated cell counter (Erma). Blood pressure was measured three times each for every volunteer in the supine position on the right arm using an automatic blood pressure monitor (Omron, M6 comfort HEM-7223-E).

\section{Urine sample preparation for RRLC-QTOF-MS}

Urine samples were diluted with an equal volume of solvent system employed for RRLC, and added with $1 \mu \mathrm{M}$ lidocaine and $5 \mu \mathrm{M}$ 5, 7-isoflavone (internal standards). 
The samples were mixed briefly and centrifuged at $5,000 \mathrm{~g}$ for $20 \mathrm{~min}$ at $4^{\circ} \mathrm{C}$ to remove precipitated protein and particulates. The supernatants were transferred to LCMS autosampler vials.

\section{RRLC-QTOFMS}

LCMS experiments were performed on an Agilent 1290 Infinity Series RRLC-MS interfaced to an Agilent 6538 Accurate-Mass Q-TOF-MS. A volume of $10 \mu \mathrm{l}$ of each sample was injected into an assembly of $\mathrm{C}_{18}$ guard column followed by a Poroshell SB-120 $(3 \times 100 \mathrm{~mm})$ $\mathrm{C}_{18}$ column of particle size 5.0 micron. The solvent system had (A) $0.1 \%$ formic in water and (B) $0.1 \%$ formic in acetonitrile: water $(80: 20)$. The gradient mode $\{\% / \min \}$ for solvent B $20 \% / 0 ; 50 \% / 18 ; 90 \% / 21$; $90 \% / 25 ; 20 \% / 29 ; 20 \% / 35\}$, with a flow rate of $0.3 \mathrm{ml} / \mathrm{min}$ was used. The mass spectrometer was operated in positive ion polarity mode with $165 \mathrm{~V}$ fragmentor voltage and ramped collision energy. The quadruple was operated in the extended dynamic range $(1700 \mathrm{~m} / \mathrm{z}, 2 \mathrm{G} \mathrm{Hz})$. To assure the mass accuracy of recorded data, continuous internal calibration was performed.

\section{Statistical analysis}

The significance of metabolite concentration differences was determined using repeated measures ANOVA with Bonferroni correction for multiple comparisons. An unpaired $t$ test was used when comparing the means of day 0 and day 14 and $p$ value less than 0.05 were considered significant. Partial least square discrimination analysis (PLS-DA) and principle component analysis (PCA) were performed using MassProfiler Professional (MPP, Agilent Technologies) and resultant discriminatory ion abundances were plotted using software Origin from Sigma Plot.

\section{Biomarkers identification}

Q-TOF-MS generate mass data with $\pm 0.05 \mathrm{mDa}$ accuracy, therefore initial identification of metabolites was done by isotopic pattern of metabolites. Thereafter, metabolites of interest were identified by comparison of the fragmentation pattern of standard and searching for fragments and parent ions in METLIN database (http://metlin.scripps.edu/), HMDB (http://www.hmdb. ca/), Massbank, Japan (http://www.massbank.jp/) were used further to assign metabolites found in the samples.

\section{RESULTS}

\section{Clinical biochemistry}

Prior to dosing on day 0 and after daily oral administration of TCJ on day $14^{\text {th }}$, blood sample were taken for clinical biochemistry (Table 1). SGPT levels were observed to be significantly reduced in HPL patients after treatment with TCJ $(\mathrm{p}<0.05)$, however, a minor reduction was observed in the HLT individuals. SGOT level significantly decreased in HLT men and non-significantly decreased in HPL patients. On day $14^{\text {th }}$, TG, TC and LDL levels were found to be significantly reduced by 28,24 and $14 \%$ in HPL patients $(\mathrm{p}<0.05)$. HDL were found to be increased in both HLT and HPL individuals $(p<0.05)$ after treatment. Uric acid levels were observed to be reduced on day $14^{\text {th }}$ but non-significantly $(\mathrm{p}<0.177)$. RBCs and platelets counts and hemoglobin levels were found to be significantly increased $(\mathrm{p}<0.05)$ on day $14^{\text {th }}$. WBCs counts, creatinine and bilirubin levels were observed to be decreased but not significantly. Treatment with TCJ also reduced total protein and albumin levels non-significantly.

\section{Highly variable metabolites in the urine}

Initial tentative identification of highly variable metabolites in the urine samples of HLT, HPL and HLT, HPL treated individual were arrived from the search of Metlin Mass Database followed by PCA (Figure 1) and PLS-DA. Further, identification of significantly decreased metabolites in HPL individuals were confirmed by comparing the MS/MS profiles of standards and tentatively identified metabolites (Table 2). Pantothenic acid, carnitine, isovalerylcarnitine, butyrylcarnitine, L-hexanoylcarnitine, 4-hydroxyisovaleric acid, acetylcarnitine, were found to be decreased on day $14^{\text {th }}$ after treatment with TCJ as compared to day 0 in HPL groups. Branched chain amino acids, leucine, L-glycyl-L-hydroxyproline, phenylalanine, tryptophan, 3-methoxytryptamine, isoleucylproline, homoserine and hippuric acid levels were recorded to be significantly increased in HPL patients as compared to the healthy individuals. However, significantly depleted levels of these metabolites were observed on day $14^{\text {th }}$ after treatment with TCJ. Significantly decreased levels of ursodeoxycholic acid $(\mathrm{p}<0.05)$, chenodeoxycholic acid $(\mathrm{p}<0.01)$ and hippuric acid $(0.05)$ were observed on day $14^{\text {th }}$.

\section{Relative quantitation of biomarkers in urine of different groups}

PLS-DA showed a number of variable metabolites which were further identified with standards. ESI-QTOFMS was used to quantify reported markers of hyperlipidemia which were also identified after PLS-D analysis of present data. Urine Samples of each volunteer, i.e. HLT, HPL and HPL treated with TCJ were subjected to quantitative analysis of selected biomarkers of HPL. A significant depletion of pantothenic acid from urine after treatment 


\begin{tabular}{|c|c|c|c|c|}
\hline \multirow[b]{2}{*}{ Parameters (Units) } & \multicolumn{2}{|c|}{ Healthy Individuals } & \multicolumn{2}{|c|}{ Hyperlipidemic Patients } \\
\hline & $\begin{array}{l}\text { Levels in Un-treated } \\
\text { Mean } \pm S D\end{array}$ & $\begin{array}{c}\text { Levels in Post-treated } \\
\text { plasma } \\
\text { Mean } \pm \text { SD }\end{array}$ & $\begin{array}{l}\text { Levels in Un-treated } \\
\text { Mean } \pm S D\end{array}$ & $\begin{array}{l}\text { Levels in Post-treated } \\
\text { Mean } \pm \text { SD }\end{array}$ \\
\hline Triglycerides $(\mathrm{mg} / \mathrm{dl})$ & $92.3 \pm 14.0$ & $58.2 \pm 13.3$ & $250 \pm 1.6$ & $180.4 \pm 17.4$ \\
\hline Total cholesterol (mg/dl) & $155.1 \pm 14.4$ & $140.2 \pm 11.5$ & $226.7 \pm 16.0$ & $180.5 \pm 12.3$ \\
\hline $\mathrm{HDL}(\mathrm{mg} / \mathrm{dl})$ & $41.1 \pm 2.5$ & $48.6 \pm 5.8$ & $12.5 \pm 6.5$ & $27.5 \pm 9.0$ \\
\hline LDL (mg/dl) & $81.5 \pm 46.1$ & $65.7 \pm 3.1$ & $143.9 \pm 11.1$ & $96.2 \pm 3.1$ \\
\hline Urea (mg/dl) & $21.5 \pm 8.9$ & $16.1 \pm 4.0$ & $18.8 \pm 1.7$ & $13.6 \pm 5.9$ \\
\hline Creatinine (mg/dl) & $1.1 \pm 0.6$ & $0.9 \pm 0.4$ & $1.35 \pm 0.4$ & $0.95 \pm 0.07$ \\
\hline Total Protein (g/dl) & $5.6 \pm 0.3$ & $6.3 \pm 0.3$ & $5.3 \pm 0.4$ & $5.0 \pm 0.5$ \\
\hline Albumin (g/dl) & $4.0 \pm 0.4$ & $3.3 \pm 1.0$ & $5.2 \pm 0.1$ & $3.9 \pm 1.2$ \\
\hline SGPT (U/L) & $28.4 \pm 12.2$ & $21.0 \pm 6.5$ & $36.0 \pm 16.3$ & $24.9 \pm 14.0$ \\
\hline SGOT (U/L) & $29.1 \pm 13.6$ & $12.0 \pm 7.5$ & $23.4 \pm 14.2$ & $20.5 \pm 16.3$ \\
\hline Uric acid $(\mathrm{mg} / \mathrm{dl})$ & $4.9 \pm 2.9$ & $4.1 \pm 1.6$ & $10.1 \pm 0.9$ & $7.7 \pm 1.9$ \\
\hline Glucose (mg/dl) & $107.0 \pm 37.0$ & $85.1 \pm 6.5$ & $124.9 \pm 18.5$ & $102.5 \pm 7.8$ \\
\hline Bilirubin $(T)(\mathrm{mg} / \mathrm{dl})$ & $1 \pm 0.1$ & $0.9 \pm 0.3$ & $1.25 \pm 0.07$ & $0.9 \pm 0.1$ \\
\hline Bilirubin (D) (mg/dl) & $0.2 \pm 0.1$ & $0.6 \pm 0.2$ & $0.25 \pm 0.2$ & $0.4 \pm 0.1$ \\
\hline Hemoglobin \% (g/dl) & $13.6 \pm 1.6$ & $14.9 \pm 1.7$ & $12.6 \pm 1.3$ & $14.2 \pm 0.6$ \\
\hline RBCs $\left(\times 10^{6}\right)$ & $4.7 \pm 0.3$ & $5.2 \pm 0.1$ & $4.6 \pm 0.6$ & $5.0 \pm 0.07$ \\
\hline WBCs $\left(x 10^{3}\right)$ & $7.3 \pm 1.0$ & $6.4 \pm 1.2$ & $7.9 \pm 0.3$ & $6.3 \pm 1.7$ \\
\hline Platelets $\left(\times 10^{3}\right)$ & $296.4 \pm 125.3$ & $313.6 \pm 85.2$ & $276.5 \pm 95.4$ & $342.5 \pm 24.7$ \\
\hline
\end{tabular}

\begin{tabular}{|c|c|c|c|c|}
\hline Name & $\begin{array}{l}\text { Retiontion } \\
\text { time (min) }\end{array}$ & $\begin{array}{l}\text { Parent ion } \\
(\mathrm{m} / \mathrm{z})\end{array}$ & Product ions $(\mathrm{m} / \mathrm{z})$ & $\begin{array}{c}\text { Regulation } \\
\text { HPL vs } \\
\text { HPL+TC }\end{array}$ \\
\hline Pantothenic acid & 10.73 & 220.12 & $202.11,184.09,142.06,124.05$ & Down \\
\hline Carnitine & 5.31 & 162.12 & 103.041 & Down \\
\hline Isovalerylcarnitine & 16.73 & 246.17 & $187.09,144.10$ & Down \\
\hline Butyrylcarnitine & 12.36 & 233.14 & $173.1,185.14,203.02,215.04$ & Down \\
\hline L-Hexanoylcarnitine & 7.70 & 260.17 & $117.06,144.11,183.11,201.12$ & Down \\
\hline 4-Hydroxyisovaleric acid & 25.72 & 118.07 & $147.02,131.04,115.02,101.02$ & Down \\
\hline Acetylcarnitine & 5.88 & 204.12 & $204.12,145.06,126.06,100.08$ & Down \\
\hline Decanoylcarnitine & 33.95 & 317.24 & $144.14,155.14,257.17$ & Down \\
\hline Leucine & 14.09 & 132.08 & 132.08 & Down \\
\hline Phenylalanine & 9.66 & 166.08 & 120.08 & Down \\
\hline Tryptophan & 14.4 & 205.08 & $188.08,146.06$ & Down \\
\hline 3-Methoxytryptamine & 18.9 & 191.12 & $174.08,159.08,143.06,131.04$ & Down \\
\hline Isoleucylproline & 5.4 & 229.13 & 132.08 & Down \\
\hline Hippuric acid & 14.8 & 179.07 & $161.6,135.06,105.02$ & Down \\
\hline Ursodeoxycholic acid & 15.08 & 393.28 & $\begin{array}{c}150.09,231.18,309.18,339.20,357.21 \\
375.22\end{array}$ & Down \\
\hline
\end{tabular}


with TCJ was observed when compared on day $14^{\text {th }}$ $(p<0.01)$ (Figure 2A). Almost 2.0 fold depletion of pantothenic acid in urine was observed on day $14^{\text {th }}$ as compared with day 0 . In other words, the levels of pantothenic acid in urine come almost equal to the HLT individuals.

Acylcarnitines were reported to be most important markers after pantothenic acid. PLS-DA shows the increased levels of several acylcarnitines in HPL urine samples. Rapid resolution chromatography resolved a number of acylcarnitines which were later on quantified. Urinary acetylcarnitine and butyryl-L-carnitine were found to be significantly depleted on day $14^{\text {th }}(\mathrm{p}<0.05)$ in patients of HPL only after treatment (Figure 2B, 2C). However, free carnitine levels were less affected with the treatment of TCJ $(p<0.15)$ (Figure 2D). On the $14^{\text {th }}$ day, 2 fold decrease in acetylcarnitine levels and 12 fold decrease in butyryl-L-carnitine levels were observed when compared with HPL patients. Other marker metabolites i.e. isovalerylcarnitine, tryptophan, ursodeoxycholic acid and phenylalanine were also found to be significantly depleted on day $14^{\text {th }}$ in HPL patients after treatment with TCJ $(\mathrm{p}<0.01)$ (Figure 3A-D).

L-Hexanoylcarnitine, 4-hydroxyisovaleric acid, two new acylcartinine, reported first time, found to be increased in HPL patients by 18 fold as compared to healthy individuals and depleted by 5 fold on day $14^{\text {th }}$. Some amino acids and their derivatives like L-glycyl-L-hydroxyproline, phenylalanine, tryptophan, isoleucylproline and hippuric acid were also observed to be increased in HPL patients and reverted back to almost healthy levels on the day $14^{\text {th }}$ after treatment with TCJ.

\section{DISCUSSION}

TCJ is the rich source of polyphenols and other medicinally important metabolites including, tinosporine, tinosporide, tinosporaside, cordifolide, cordifol, heptacosanol, clerodane furanoditerpene, diterpenoid furanolactone tinosporidine, palmatine, tembertarine and $\beta$-sitosterol. ${ }^{5}$ Crude extracts and purified metabolites from the plant have been reported for various kinds of medicinal properties. Several metabolite of T. cordifolia such as proanthocyanidins, epicatechin, cordioside, cordiofolioside A, ferulic acid, magnoflorine, columbin etc. are reported to be absorbed and act as in vivo antioxidant. ${ }^{12,13}$ Columbamine from the plant was reported for the AChE inhibitory activity. ${ }^{14}$ Berberine, and its analogue alkaloids from the plant have been reported for antihyperlipidemic properties. ${ }^{15}$ Compounds isolated from the plants were also reported to increase glucose uptake activity by increasing GLUT-4 expression. ${ }^{9}$ Moreover,

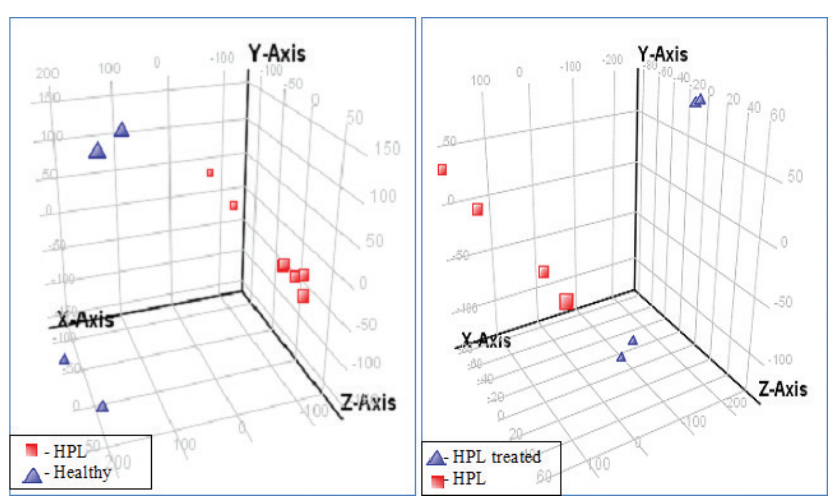

Figure 1: In PCA plots square and triangles represent treated and HPL spatial relation between the molecular features were represented along with $X, Y$ and $Z$ axis. ( $A$ ) represents the spatial relation between the metabolites of healthy (Blue triangles)

and HPL individuals (Red, squares) where variability along the $X, Y$, and $Z$ axis are $26.30 \%, 17.11 \%, 9.63 \%$ respectively, (B) represents the spatial relation between the metabolites of HPL (Red, squares) and HPL treated (Blue triangles) with TCJ individual where variability along the $X$-axis is 42.44 and along the $Y, Z$ axis is $18.38 \%, 9.89 \%$ respectively.
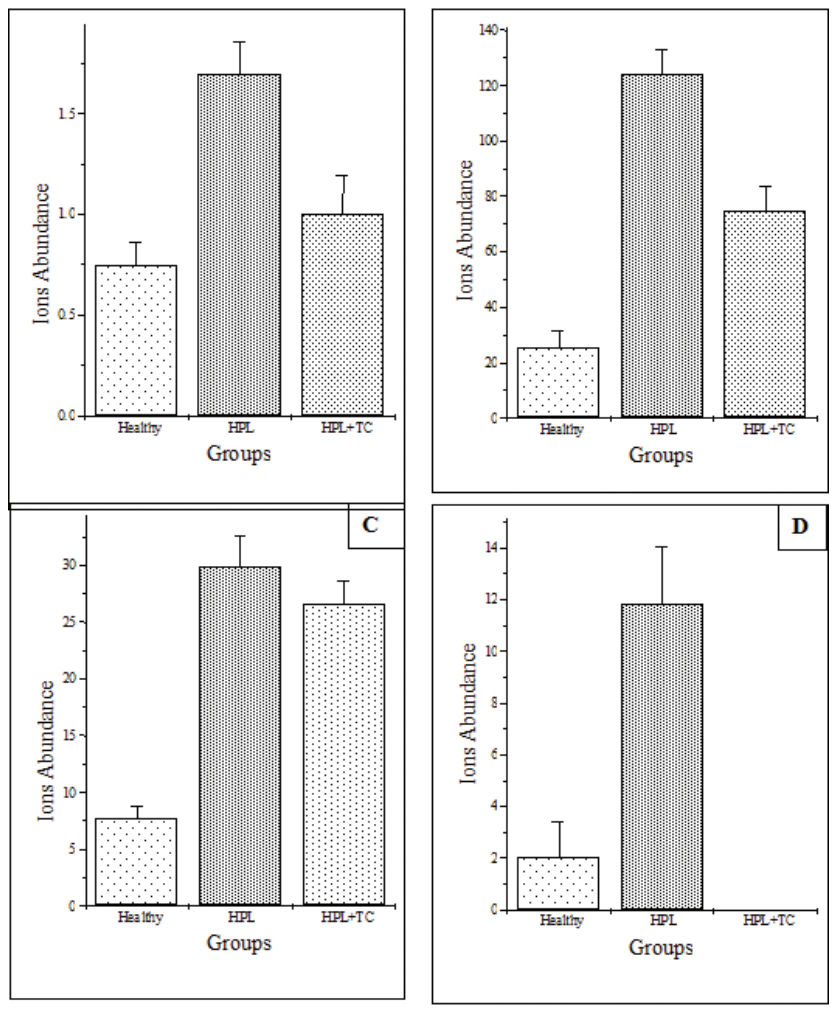

Figure 2: Urinary $(A)$ pantothenic acid $(P<0.001)$, (B) acetylcarnitine $(p<0.005),(C)$ carnitine $(p<0.15)$, (D) butyryl-L-carnitine $(p<0.001)$ in healthy, HPL and HPL individuals treated with TCJ (on day 14) ( $p=0.01)$. 


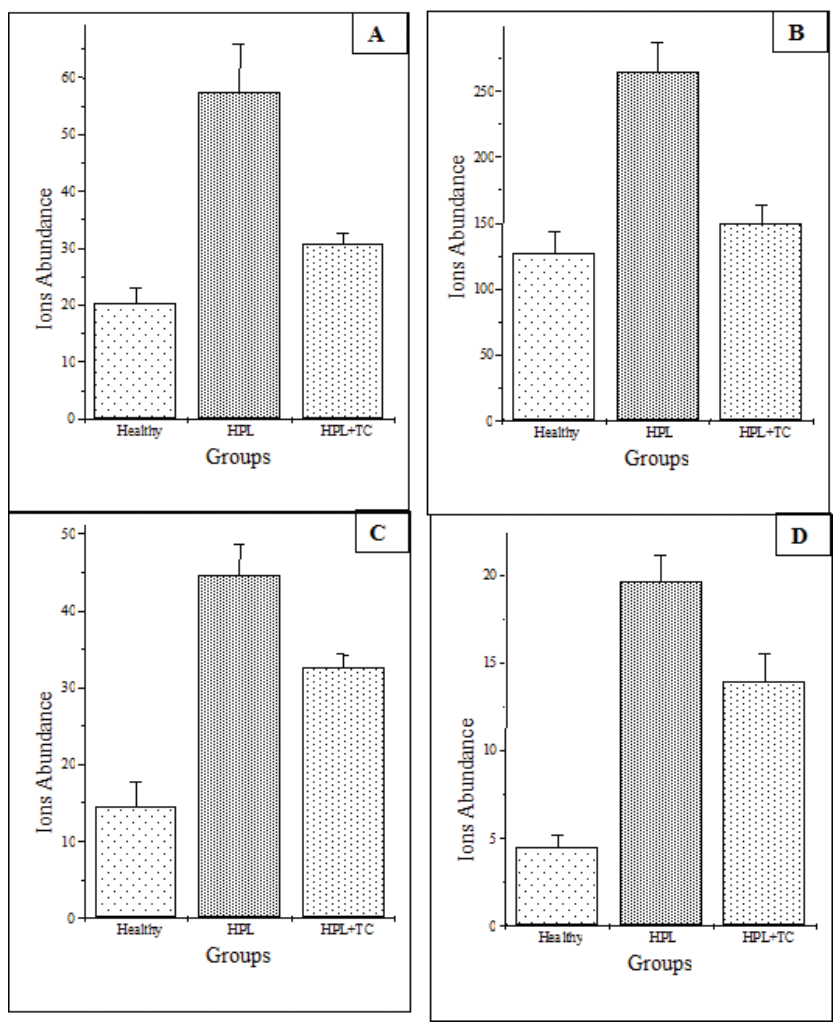

Figure 3: Urinary $(A)$ isovalerylcarnitine $(p<0.05)$, (B) tryptophan $(p<0.01)(C)$ ursodeoxycholic acid $(p<0.01)$ and (D) phenylalanine $(p<0.01)$ in healthy, HPL and HPL individuals treated with TCJ (on day 14$)(p<0.01)$.

T. cordifolia is well known for in vivo immunomodulatory effects, ${ }^{16}$ however, immunomodulatory compounds from the plant, yet to be identified.

A number of studies have been conducted to explore the medicinal properties of crude extracts and isolated pure compounds. Major emphasis in previous studies was given to in vitro bio-activities of the plant. However, conducted clinical trials also showed the clinical efficacy of plant. The major drawback of most the studies is the lack of in vivo correlation of medicinal efficacy and lack of justification for mechanism of action. As several studies have proven that in vitro and in vivo efficacy of extracts and isolated pure compounds may be different, therefore, it is essential to study the efficacy of molecule in vivo before the claim of their medicinal efficacy. Additionally, studies of mechanism of action will provide fate in the natural extracts or pure compounds isolated from plant. In present study, in vivo effects of TCJ were analyzed on the human urinary biomarkers of HPL using a metabolomics approach consisting of RRLCESI-QTOFMS, PCA and PLS-DA. Increased fatty acid catabolism is associated with increased activation of PPAR $\alpha$ hence commonly targeted for the treatment of HPL. Therefore, urinary biomarker as described earlier in hyperlipidemics were analysed from the discriminated data. ${ }^{11}$ Urinary pantothenic acid and acylcarnitines has been reported to be useful biomarkers of PPAR $\alpha$ induced fatty acid beta-oxidation in humans. Significant depletion of both pantothenic acid ( $>3$-fold) and acetylcarnitine $\left(>12\right.$-fold), on day $14^{\text {th }}$ in TCJ treated HPL patients was observed in PLS-DA. PPAR $\alpha$ activation increases synthesis of pantothenate kinase and proteins involved in the transport and synthesis of acylcarnitines. Analysis showed that TCJ activated the PPAR $\alpha$ which regulate the $\beta$-fatty acid oxidation. It was further supported by decreased levels of serum cholesterol, triglycerides, uric acid by day $14^{\text {th }}$ in HPL treated with TCJ. In vitro activation of PPAR $\alpha$ by T. cordifolia has been reported earlier. ${ }^{9}$ This study confirms the in vitro result and validates in vivo activity of TCJ as PPAR $\alpha$ modulator.

\section{CONCLUSION}

Recent advances in LC-MS based metabolomics have created the potential to measure the levels of numerous metabolites even in complex human biological samples. Metabolomic profiling by label-free LC-MS methods to find candidate metabolic biomarkers for early detection of complex disease and identification of metabolites by spectral matching are relatively economical, simple and easily repeatable approaches are effectively used here to predict few probable biomarkers of hyperlipidemia in human earlier reported in mouse models. Metabolomics has also opened the avenue to study the mechanism of complex herbal drugs by quantifying the reported and unknown biomarkers of diseases using PLS-DA. Analysis of HPL biomarkers by PLS-DA, it was found that TCJ acts as agonist of PPAR $\alpha$ and PPAR- $\gamma$ which indirectly or directly regulate LDLR and GLUT-4. The current study also supports the earlier mouse model studies as pantothenic acid, short chain acylcarnitines and branched chain amino acids are also found to be biomarkers of hyperlipidemia in the human beings. Main impact of study is to reveal the targets of complex extracts which may lead to discovery of novel non-toxic compound(s) using in vitro assays to treat hyperlipidemic patients.

\section{ACKNOWLEDGEMENT}

Authors wish to acknowledge the CCRAS, Ministry of AYUSH, Govt of India for supporting this work morally.

\section{CONFLICT OF INTEREST}

Both authors (RD, ARS) declare that they have no conflicts of interest involving this work. 


\section{ABBREVIATION USED}

TCJ: Tinospora cordifolia juice; RRLC-QTOFMS: Rapid resolution liqid chromatography-quadruple time of flight mass spectrometer; TC: Total cholesterol; TG: Triglyceride; LDL: Low density lipoproteins; HDL: High density lipoproteins; PPAR- $\alpha$ : Peroxisome proliferator-activated receptor alpha; HPL: Hyperlipidemic; HLT: Healthy; SGOT: Serum glutamic oxaloacetic transaminase; SGPT: Serum glutamic-pyruvic transaminase; PLS-DA: Partial least square discrimination analysis; PCA: Principle component analysis; AChE: Acetylcholinesterase.

\section{REFERENCES}

1. Saha S, Ghosh S. Tinospora cordifolia: One plant, many roles. Anc Sci Life. 2012;31(4):151-9. http://dx.doi.org/10.4103/0257-7941.107344; PMid:23661861 PMCid:PMC3644751.

2. Sharma V, Gupta R, Sharma S. Hypoglycemic and hypolipidemic activity of Tinospora cordifolia root extract on aflatoxin B1-induced toxicity in mice. Int $\mathrm{J}$ Res Pharm Sci. 2011;2(4):585-9.

3. Meshram A, Bhagyawant SS, Gautam S, Shrivastava N. Potential Role of Tinospora Cordifolia in Pharmaceuticals. World Journal of Pharmacy and Pharmaceutical Sciences. 2013;2(6):4615-25.

4. Sharma V, Gupta R, Mishra N, Sharma S. Influence of Tinospora cordifolia Root Extract Supplementation on Hematological and Serological Parameters of Male Mice Exposed to Aflatoxin B1. International Journal of Pharmacology. 2011;7:659-63. http://dx.doi.org/10.3923/ijp.2011.659.663.

5. Choudhary N, Siddiqui MB, Azmat S, Khatoon S. Tinospora Cordifolia: Ethnobotany, Phytopharmacology and Phytochemistry Aspects. International Journal of Pharmaceutical Sciences and Research. 2013;4(3):891-9.

6. Singh SS, Pandey SC, Srivastava S, Gupta VS, Patro B, Ghosh AC. Chemistry and Medicinal Properties of Tinospora Cordifolia (Guduchi). Indian Journal of Pharmacology. 2003;35:83-91.
7. Kong WJ, Wei J, Zuo ZY, Wang YM, Song DQ, You XF et al. Combination of simvastatin with berberine improves the lipid-lowering efficacy. Metabolism. 2008; 57(8):1029-37. http://dx.doi.org/10.1016/j.metabol.2008.01.037; PMid:18640378.

8. Vasanthi HR, Kannan SM. Palmatine a Novel Anti-adipogenic and Antidiabetic Alkaloid from an Indian Medicinal Plant Tinospora cordifolia. The FASEB Journal. 2012;26:112-8.

9. Sangeethaa MK, Mohana Priyab CD, Vasanthia HR. Anti-diabetic property of Tinospora cordifolia and its active compound is mediated through the expression of Glut-4 in L6 myotubes. Phytomedicine. 2013;20:246-8. http:// dx.doi.org/10.1016/j.phymed.2012.11.006; PMid:23290487

10. Sharma R, Amin H, Galib, Prajapati PK. Antidiabetic claims of Tinospora cordifolia (Willd.) Miers: critical appraisal and role in therapy. Asian Pacific Journal of Tropical Biomedicine. 2015;5(1):68-78. http://dx.doi.org/10.1016/ S2221-1691(15)30173-8.

11. Patterson AD, Slanař O, Krausz KW, Li F, Höfer CC, Perlík F et al. Human Urinary Metabolomic Profile of PPARa Induced Fatty Acid $\beta$-Oxidation. J Proteome Res. 2009;8(9):4293-300. http://dx.doi.org/10.1021/pr9004103; PMid:19569716 PMCid:PMC2760252.

12. Bhawya D, Anilakumar KR. In vitro Antioxidant Potency of Tinospora cordifolia (gulancha) in Sequential Extracts. International Journal of Pharmaceutical \& Biological Archives. 2010;1(5):448-56.

13. Haque Aminul Md, Islam Ashraful SM, Mohammad S. Antimicrobial, Cytotoxicity and Antioxidant Activity of Tinospora crispa. Journal of Pharmaceutical and Biomedical Sciences. 2011;13(12):1-4.

14. Yusoff M, Hamid H, Houghton P. Anticholinesterase inhibitory activity of quaternary alkaloids from Tinospora crispa. Molecules. 2014;19(1):1201-11. http://dx.doi.org/10.3390/molecules19011201; PMid:24448061.

15. Vuddanda PR, Chakraborty S, Singh S. Berberine: a potential phytochemical with multispectrum therapeutic activities. Expert Opin Investig Drugs. 2010;19(10): 1297-307. http://dx.doi.org/10.1517/13543784.2010.517745; PMid:20836620.

16. Sinha K, Mishra NP, Singh J, Khanuja SPS. Tinospora cordifolia (Guduchi), a reservoir plant for therapeutic applications: A Review. Indian Journal of Traditional Knowledge. 2004;3(3):257-70.

\section{SUMMARY}

- Tinospora cordifolia (Willd.) Miers juice (TCJ) possesses hypolipidemic properties.

- TCJ treatment depleted TC, TG and LDL levels in HPL patients.

- It also depleted urinary levels of BCAA, pantothenic acid, hippuric acid and carnitine, biomarkers of PPAR-ם.

- TCJ was found to regulate the lipid metabolism through PPAR- $\alpha$ activation. 